\title{
IRREDUCIBLE REPRESENTATIONS OF OPERATOR ALGEBRAS
}

\author{
I. E. SEGAL
}

1. Introduction. In this paper we investigate the representation on (not necessarily separable) Hilbert spaces of a suitably closed algebra of operators on a Hilbert space, with regard to the existence of irreducible representations and the connection between representations and states of the algebra (we use the term "state" to mean a complexvalued linear function on the algebra which is real and non-negative on positive semi-definite operators, and which satisfies a certain normalizing condition-more commonly, this is called an expectation value in a state). Our results are, first, that there is a mutual correspondence between "normal" representations (those for which there is an element of the Hilbert space whose transforms under the representation operators span the space) and states of the algebra, in which irreducible representations correspond to pure states (a state is pure if it is not a linear combination with positive coefficients of two other states), and second, that there exists a complete set of irreducible representations for a given algebra (or alternatively, a complete set of pure states).

The operator algebras which we consider are assumed to be: (1) closed in the uniform topology (in which the distance between two operators is defined as the bound of their difference), (2) self-adjoint (that is, if an operator is in the algebra then so is its adjoint); we call such an algebra a $C^{*}$-algebra. By a representation of a $C^{*}$-algebra $\mathcal{A}$ we mean a function $\phi$ on $\mathcal{A}$ to the collection of bounded operators on a Hilbert space which is an algebraic homomorphism, continuous, and such that for $U \notin \mathcal{A}, \phi\left(U^{*}\right)=(\phi(U))^{*}$, the asterisks denoting adjoints. The representation is called irreducible if the Hilbert space on which $A$ is represented contains no nontrivial closed invariant subspace. A collection of representations of $A$ is called complete if the only element of $\mathcal{A}$ which is mapped into zero by every representation is zero.

Our results make possible a broader and more rigorous treatment of certain parts of quantum mechanics, particularly of the principle that the spectral values of an observable are determined by the behavior of the observable in the irreducible representations of the operator algebra describing the physical system in question. The original mathematical model for the observables in quantum theory was the

Presented to the Society, August 23, 1946; received by the editors May 3, 1946. 
class of all self-adjoint elements of the algebra $\mathcal{C}$ of all bounded operators on a Hilbert space (frequently unbounded operators were included, but these could be treated in terms of bounded operators, and did not affect the states or representations of the system of observables). For a variety of reasons, however, it seems desirable to consider the collection of self-adjoint elements in less restricted algebras of operators as a possible model for the observables, the most notable reason being: (a) the lack of a compelling physical reason why every self-adjoint operator should correspond to an observable, (b) the serious mathematical difficulties in quantum electrodynamics, (c) in a postulational treatment of quantum mechanics which we hope to present elsewhere, the collection of self-adjoint elements of a $C^{*}$-algebra satisfies our axioms for the system of observables. The concept of pure state was introduced by von Neumann and Weyl (independently) in a quite general fashion and in the case of the algebra $\mathcal{C}$ just mentioned it was shown that an element of the Hilbert space of norm unity (a wave function) gave rise to a pure state. In more general systems the question of the existence of pure states was left open, but we should mention that our proof of this existence is based on a theorem of Krein, Milman, and Shmulyan [4] ${ }^{1}$ which was proved fairly recently.

Our interest in operator algebras grew out of our investigations of group algebras of locally compact groups, a group algebra being isomorphic with a certain self-adjoint operator algebra. Applying our theorem in the case of the latter algebra, we obtain as a corollary a recent result of Gelfand and Rykov, to the effect that an arbitrary locally compact group has a complete set of strongly continuous irreducible unitary representations (which we show can be assumed to be derived from representations of the $C^{*}$-algebra which is the uniform closure of that algebra). Our procedure here is somewhat similar to that much used in the theory of finite groups and also in [6], in which it is first shown that an algebra built on the group is, in some sense, semi-simple, and therefore has a complete set of irreducible representations, from which the existence of the desired group representations may be concluded.

Our proofs make use of devices previously employed by Gelfand and Neumark [2], Gelfand and Rykov [3], and by ourselves [6]. Like Gelfand and Rykov we utilize the theorem of Krein, Milman, and Shmulyan mentioned above; unlike them we make no use of positive definite functions, in deriving the group-theoretical corollary mentioned in the preceding paragraph.

${ }^{1}$ Numbers in brackets refer to the references cited at the end of the paper. 
We are indebted to $\mathrm{W}$. Ambrose for suggestions which resulted in a significant extensions of our theory and in important simplifications in the proofs.

2. Definitions. Let $A$ be a $C^{*}$-algebra, by which we mean a uniformly closed, self-adjoint algebra of bounded operators on a Hilbert space. In other words, if $U_{n}(n=1,2, \cdots), V$, and $W$ are elements of $\mathcal{A}$, and if $\lim _{n}\left\|U_{n}-U\right\|=0$, where $\|T\|=$ L.U.B. ${ }_{(x, x=1)}(T x, T x)^{1 / 2}$, and $(x, y)$ is the Hilbert space inner product, then the following operators are all in $\mathcal{A}: U, V W, V+W, V^{*}$, and $\alpha V$, for an arbitrary complex number $\alpha$. We use the term Hilbert space for a space over the complex field which satisfies the usual axioms, except that the axiom of separability is not necessarily satisfied. The complex conjugate of an expression is denoted by enclosing it in parentheses and using the right superscript $c$.

Definition 1. $A$ state of $\mathcal{A}$ is a complex-valued linear function $\omega$ on $\mathcal{A}$ such that $\omega\left(U^{*}\right)$ is the complex conjugate of $\omega(U), \omega(U) \geqq 0$, if $U$ is positive semi-definite and such that L.U.B. $\|_{\| \rrbracket ! 1} \omega\left(U U^{*}\right)=1$. A pure state is one which is not a linear combination with positive coefficients of two other states.

For example, if $x$ is an element of the Hilbert space of norm unity, setting $\omega(U)=(U x, x)$ defines a state. In the special case that $\mathcal{A}=C$, this state is pure, but in general the state is not pure. We observe that if $\mathcal{A}$ contains the identity operator $I$, then the condition L.U.B. $\| U \rrbracket \leqq \omega\left(U U^{*}\right)=1$ is equivalent to the simpler condition $\omega(I)$ $=1$, because if $\|U\| \leqq 1$, then $I-U U^{*}$ is positive semi-definite.

Definition 2. A representation of $\mathcal{A}$ on a Hilbert space $\mathfrak{H C}$ is a mapping $\phi$ on $\mathcal{A}$ to the collection of bounded linear operators on $3 C$ such that for general $U$ and $V$ in $A$ and an arbitrary complex number $\alpha: \phi(U+V)=\phi(U)+\phi(V), \phi(U V)=\phi(U) \phi(V), \phi\left(U^{*}\right)=(\phi(U))^{*}$, $\phi(\alpha U)=\alpha \phi(U)$. A representation is called normal if there exists an element $x$ in $\mathfrak{H}$ such that the span of ${ }^{2}[\phi(U) x \mid U \in \mathcal{A}]$ is $\mathfrak{K C}$; if $x$ is of norm unity, the function $\omega$ defined by $\omega(U)=(\phi(U) x, x)$ is ca lled a normalizing function. A representation is called irreducible if ther exists no proper closed invariant subspace of $\mathfrak{T}$ other than the nullspace. Two representations are called equivalent if there is a unitary transformation on one Hilbert space to the other which maps one representation into the other.

Definition 3. A collection of states of $\mathcal{A}$ is called complete if there is no element of $\mathcal{A}$ except zero which vanishes in every state of the

\footnotetext{
${ }^{2}$ The notation $[f(a) \mid a \in S]$ is used to denote the range of values of $f(a)$ as $a$ varies over $S$.
} 
collection. A collection of representations of $\mathcal{A}$ is called complete if no element of $\mathcal{A}$ except zero is mapped into zero by every representation in the collection.

3. The relation between states and normal representations. In the present section we show that there is a natural mutual correspondence between states and normal representations of a $C^{*}$-algebra $A$, in which pure states and irreducible representations correspond. This is interesting from the standpoint of quantum theory because it shows the general connection between two concepts of importance in that theory which have been independently introduced.

THEOREM 1. A normalizing function of a normal representation of a $C^{*}$-algebra on a Hilbert space is a state of the algebra. Conversely, a state of the algebra is the normalizing function of some normal representation, and this representation is uniquely determined by the state, within equivalence. The state is pure if and only if the corresponding representation is irreducible.

The proofs are somewhat simpler in the case that the $C^{*}$-algebra contains the identity operator, but it is essential for applications to group representations to consider the more general case in which the identity is not necessarily contained in the algebra. In order to discuss this more general case we prove a lemma to the effect that an arbitrary $C^{*}$-algebra contains a kind of approximate identity. This approximate identity plays roughly the same part in our proofs as the identity would, in case the treatment were limited to algebras containing the identity.

Definition 4. An approximate identity of a $C^{*}$-algebra $A$ is a Moore-Smith directed system $V_{\mu}$ of elements of $\mathcal{A}$ such that $\left\|V_{\mu}\right\| \leqq 1$ and $\lim _{\mu} V_{\mu} U=U$ for every $U$ in $\mathcal{A}$.

Lemma 1.1. A $C^{*}$-algebra contains an approximate identity.

Let $\mu$ be a general finite subset of the $C^{*}$-algebra $\mathcal{A}$. It is plain that the $\mu$ 's form a directed system relative to set inclusion. For $\mu=\left[U_{1}, \cdots, U_{n}\right]$, put $W=\sum_{i=1}^{n} U_{i} U_{i}^{*}$, and define $V_{\mu}=W(\epsilon I+W)^{-1}$, where $\epsilon=n^{-1}$. Then $V_{\mu} \in \mathcal{A}$ and $\left(V_{\mu}-I\right) W\left(V_{\mu}-I\right)^{*}=\epsilon^{2} W(\epsilon I+W)^{-2}$. If we write $X \leqq Y$ for SA (self-adjoint) operators $X$ and $Y$ if $Y-X$ is positive semi-definite, it is clear that $\epsilon^{2} W(\epsilon I+W)^{-2} \leqq \epsilon I / 2$. Hence

$$
\sum_{i=1}^{n}\left[\left(V_{\mu}-I\right) U_{i}\right]\left[\left(V_{\mu}-I\right) U_{i}\right]^{*} \leqq \epsilon I / 2,
$$

and it follows that each term in the sum is less than or equal to $\epsilon I / 2$. 
This implies that $\left\|\left(V_{\mu}-I\right) U_{i}\right\| \leqq 1 / 2 n$, and therefore $\lim _{\mu} V_{\mu} U=U$ for arbitrary $U \in \mathcal{A}$, completing the proof of the lemma.

Now we show that a normalizing function of a normal representation $\phi$ of an algebra $\mathcal{A}$ on a Hilbert space $\mathfrak{H}$ is a state of $\mathcal{A}$. Except for the condition

$$
\text { L.U.B. } \omega\left(U U^{*}\right)=1 \text {, }
$$

where $\omega(U)=(\phi(U) z, z)$, and $z$ is an element of $\mathcal{H C}$ such that $[\phi(U) z \mid U \in \mathcal{C} A]$ spans $\mathfrak{H}$, it is trivial to verify that the conditions of Definition 1 are satisfied. We begin the verification of (1) by showing that the bound of $\phi$ is unity. ${ }^{3}$ Let $\mathcal{A}^{\prime}$ be the algebra generated by $\mathcal{A}$ and the identity operator $I$. It is readily seen that $A^{\prime}$ is uniformly closed and self-adjoint. We extend the representation $\phi$ to $\mathcal{A}^{\prime}$ by defining: $\phi^{\prime}(\alpha I+U)=\alpha \tilde{I}+\phi(U)$, where $U$ is an arbitrary element of $\mathcal{A}$ and $I$ is the identity operator on $\mathcal{H}$. Clearly, $\phi^{\prime}$ is a representation of $\mathcal{A}^{\prime}$ on $\mathcal{H}$. Now if $\|U\| \leqq 1$, then $I-U U^{*}$ is positive semi-definite, and therefore has the form $V V^{*}$ for some $V \in \mathcal{A}$. Since $\phi\left(I-U U^{*}\right)=\phi(V)(\phi(V))^{*}$, it follows that $\phi\left(U U^{*}\right) \leqq I$, and $\left\|\phi\left(U U^{*}\right)\right\| \leqq 1$. Since $\left\|\phi\left(U U^{*}\right)\right\|=\|\phi(U)\|^{2}$, this proves $\|\phi(U)\|$ $\leqq 1$. Putting L.U.B. $n U \llbracket \leq 1\|\phi(U)\|=M$, it follows easily that L.U.B. $\left\|U_{\| \leq 1}\right\| \phi\left(\left[U U^{*}\right]^{1 / 4}\right) \|=M^{1 / 2} ;$ and since $\left(U U^{*}\right)^{1 / 4} \in \mathcal{A}$ when $U \in \mathcal{A}$, it results that $M^{1 / 2} \leqq M$. It is trivial to conclude that L.U.B. $B_{\|U\| \leq 1}\|\phi(U)\|=1$. Clearly,

$$
\underset{\|U\| \leqq 1}{\text { L.U.B. }} \omega\left(U^{*} U\right)=\underset{\|U\| \leqq 1}{\text { L.U.B. }}(\phi(U) z, \phi(U) z) \leqq 1 .
$$

To complete the proof, let $V_{\mu}$ be an approximate identity of $\mathcal{A}$. It is plain that $\lim _{\mu}\left(\phi\left(V_{\mu}\right) z, \phi(U) z\right)=(z, \phi(U) z)$ for $U \in \mathcal{A}$. Now $\left\|\phi\left(V_{\mu}\right) z\right\|$ $\leqq 1$ because $\left\|V_{\mu}\right\| \leqq 1$, and $[\phi(U) z \mid U \in \mathcal{A}]$ is dense in $\mathcal{H}$. It follows easily that $\lim _{\mu}\left(\phi\left(V_{\mu}\right) z, x\right)=(z, x)$ for arbitrary $x$ in $\mathcal{H}$. Substituting $x=z$ and applying Schwarz' inequality to $\left(\phi\left(V_{\mu}\right) z, z\right)$, it follows that

$$
(z, z) \leqq \lim _{\mu} \sup \left(\phi\left(V_{\mu}\right) z, \phi\left(V_{\mu}\right) z\right)^{1 / 2},
$$

which implies L.U.B. ${ }_{\mu}\left(\phi\left(V_{\mu}\right) z, \phi\left(V_{\mu}\right) z\right) \geqq 1$, and therefore L.U.B. $\| U \rrbracket \leqq 1$ $\omega\left(U^{*} U\right) \geqq 1$. Combining the two inequalities completes the proof that $\omega$ is a state.

Now we show that a state $\omega$ of $\mathcal{A}$ is a normalizing function for some normal representation of $\mathcal{A}$, using a procedure due in part to Gelfand and Neumark [2]. Set $K$ for the class of elements $U$ of $\mathcal{A}$ such that

${ }^{3}$ We are indebted to G. W. Mackey for the observation that a representation of a $C^{*}$-algebra is necessarily continuous. 
$\omega(V U)=0$ for every $V \in \mathcal{A}$. It is easy to verify that $K$ is a left ideal. We denote the residue class of an operator modulo $K$ by superposing a bar, and define $(\bar{U}, \bar{V})=\omega\left(V^{*} U\right)$, for arbitrary elements of $\mathcal{A}, U$ and $V$. To see that this is a unique definition, observe that $\omega\left(\left(V+W_{1}\right)^{*}\left(U+W_{2}\right)\right)=\omega\left(V^{*} U\right)+\omega\left(W_{1}^{*} U\right)+\omega\left(V^{*} W_{2}\right)+\omega\left(W_{1}^{*} W_{2}\right) ;$ and if $W_{1}$ and $W_{2}$ are in $K$, then $\omega\left(W_{1}^{*} U\right)=\left(\omega\left(U^{*} W_{1}\right)\right)^{c}=0, \omega\left(V^{*} W_{2}\right)$ $=0=\omega\left(W_{1}^{*} W_{2}\right)$. That is, $\omega\left(V^{*} U\right)$ is actually a function of $\bar{U}$ and $\bar{V}$.

The function $(\bar{U}, \bar{V})$, with $\bar{U}$ and $\bar{V}$ ranging over the quotient linear space $A / K$ of $A$ modulo $K$, has the usual properties of an inner product. Specifically, it is linear in $\bar{U}$, semi-linear in $\bar{V}$, is taken into its complex conjugate by interchange of $\bar{U}$ and $\bar{V}$, and is positive definite; these facts are quite evident except for the positive definiteness, which we now prove. Since the usual proof of Schwarz' inequality in a Hilbert space makes no use of completeness,

$$
|(\bar{U}, \bar{V})| \leqq(\bar{U}, \bar{U})^{1 / 2}(\bar{V}, \bar{V})^{1 / 2} .
$$

Hence, if $(\bar{U}, \bar{U})=0, \omega\left(V^{*} U\right)=0$ for all $V \in \mathcal{A}$, which shows that $\bar{U}=0$. Now we complete $A / K$ to a Hilbert space with respect to the given inner product. First, $\mathcal{A} / K$ may be regarded as a linear metric space with the norm $\|\bar{U}\|=(\bar{U}, \bar{U})^{1 / 2}$ (the usual proof of the triangle inequality for Hilbert space makes no use of completeness). Second, $(\bar{U}, \bar{V})$ is plainly a uniformly continuous function on $(\mathcal{A} / K) \times(\mathcal{A} / K)$, and therefore its domain of definition can be extended to be $Z \times \mathcal{Z}$, where $Z$ is the completion of $A / K$, in such a way that it remains uniformly continuous. This extension is unique. It is easy to verify that $Z$ is a Hilbert space relative to this extended inner product.

For $U$ in $A$ we set $\phi_{0}(U)$ for the operator defined on $A / K$ by $\phi_{0}(U) \bar{V}=\eta(U V) ;^{4}$ this definition is unique, $K$ being a left ideal. Now we show that $\phi_{0}(U)$ is a bounded operator on $A / K$, and may therefore be extended in a continuous fashion to $Z$. Since $\|U\|^{2} I-U^{*} U \geqq 0$, $V^{*}\left(\|U\|^{2} I-U^{*} U\right) V \geqq 0$, where $U$ and $V$ are arbitrary elements of $\mathcal{A}$. Hence $\omega\left(V^{*} U^{*} U V\right) \leqq\|U\|{ }^{2} \omega\left(V^{*} V\right)$, from which it is plain that $\left(\phi_{0}(U) \bar{V}, \phi_{0}(U) \bar{V}\right) \leqq\|U\|^{2}(\bar{V}, \bar{V})$ showing that: (a) $\|\phi(U)\| \leqq\|U\|$, (b) $\phi$ is a representation of $\mathcal{A}$ on $Z$, (c) this representation is adjointpreserving, because

$$
(\phi(U) \bar{V}, \bar{W})=\omega\left(W^{*} U V\right)=\left(\bar{V}, \phi\left(U^{*}\right) \bar{W}\right),
$$

$W$ being arbitrary in $\mathcal{A}$.

To see that the representation is normal, let $\left\{V_{\mu}\right\}$ be an approxi-

${ }^{4}$ We introduce a function $\eta$ defined by $\eta(U)=\bar{U}$. 
mate identity for $A$ and observe that $\lim _{\mu}\left(\bar{W}, \bar{V}_{\mu}{ }^{*}\right)=\omega(W)$ for $W \in \mathcal{C} A$. It follows easily that $\bar{V}_{\mu}{ }^{*}$ is weakly convergent, and Hilbert space being complete in the weak neighborhood topology, there exists an element $z$ in $Z$ such that $(\bar{W}, z)=\omega(W), W \in \mathcal{A}$. For arbitrary $X \in \mathcal{A}$,

$$
(\bar{X}, \phi(W) z)=\left(\bar{W}^{*} X, z\right)=\omega\left(W^{*} X\right)=(\bar{X}, \bar{W}),
$$

showing that $\phi(W) z=\bar{W}$, and that the representation is normal. A normalizing functional is plainly $(\phi(U) z, z)=\omega(U)$, and the proof that a state of $\mathcal{A}$ is a normalizing function for some normal representation of $\mathcal{A}$ is complete.

Our next step is to show the essential uniqueness of the representation for which a given state is a normalizing function: Let $\phi^{\prime}$ be a normal representation of $\mathcal{A}$ on a Hilbert space $Z^{\prime}$ with normalizing function $\omega(U)=\left(\phi(U) z^{\prime}, z^{\prime}\right), z^{\prime}$ being an element of $Z^{\prime}$ such that $\left[\phi(U) z^{\prime} \mid U \in \mathcal{C} A\right.$ is dense in $Z^{\prime}$. We shall show that the preceding $\phi$ and $\phi^{\prime}$ are equivalent. To do this we must exhibit a unitary transformation on $Z$ to $Z^{\prime}$ which takes $\phi$ into $\phi^{\prime}$. This transformation $\theta$ is defined as follows. For $\bar{U} \in \mathcal{Z}, \theta(\bar{U})=\phi^{\prime}(U) z^{\prime}$-this is a unique definition because if $\bar{U}=\bar{V}, \omega\left(W^{*} U\right)=\omega\left(W^{*} V\right)$ for $W \in \mathcal{A}$, which implies $\left(\phi^{\prime}(U) z^{\prime}, \phi^{\prime}(W) z^{\prime}\right)=\left(\phi^{\prime}(V) z, \phi^{\prime}(W) z^{\prime}\right)$; hence $\left(\phi^{\prime}(U) z^{\prime}, x^{\prime}\right)$ $=\left(\phi^{\prime}(V) z^{\prime}, x^{\prime}\right)$ for $x^{\prime} \in Z^{\prime}$, and $\phi^{\prime}(U) z^{\prime}=\phi^{\prime}(V) z^{\prime}$. Moreover, $\theta$ is isometric, because

$$
(\bar{U}, \bar{U})=\omega\left(U^{*} U\right)=\left(\phi(U) z^{\prime}, \phi(U) z^{\prime}\right)=(\theta(\bar{U}), \theta(\bar{U})) .
$$

On the other hand, the range of $\theta$ (as $\bar{U}$ varies over $A / K$ ) is clearly dense in $Z^{\prime}$. It follows that there is a unique isometric extension of the definition of $\theta$ from the domain $A / K$ to the domain $\mathcal{Z}$, and that this extension has the range $Z^{\prime}$, and hence is unitary. Now $\phi$ and $\phi^{\prime}$ are equivalent provided $\theta \phi(U) \theta^{-1}=\phi^{\prime}(U), U \in \mathcal{A}$. Since $\theta \phi(U) \theta^{-1}$ and $\phi^{\prime}(U)$ are bounded operators, it is sufficient to check this equality on a dense domain, such as $\left[\phi(U) z^{\prime} \mid U \in \mathcal{A}\right]$. This check proceeds as follows:

$$
\theta \phi(U) \theta^{-1} \phi^{\prime}(V) z^{\prime}=\theta \phi(U) \bar{V}=\theta \eta(U V)=\phi^{\prime}(U V) z^{\prime}=\phi^{\prime}(U) \phi^{\prime}(V) z^{\prime} .
$$

We prove next that the representation corresponding to a pure state is irreducible, employing here and in the following paragraph a method similar to that used by Gelfand and Rykov [3]. As the hypothesis of an indirect proof, assume that $\omega$ is pure and that the proper nonzero closed linear subspace $S$ of $Z$ is invariant. If we put $S \perp$ for the orthogonal complement of $\mathcal{S}$, it is plain that if $x \in \mathcal{S}$ and $y \in \mathcal{S} \perp$,

$$
(x, \phi(U) y)=\left(\phi\left(U^{*}\right) x, y\right)=0,
$$


showing that $\delta \perp$ is invariant. It follows readily that the projection $P$ on $S$ commutes with $\phi(U)$ for every $U \in \mathcal{C}$. If we define $\sigma(U)=(\phi(U) P z, P z)$ and $\tau(U)=(\phi(U)(\tilde{I}-P) \boldsymbol{z},(\tilde{I}-P) z)$, it is easy to verify that $\omega=\sigma+\tau$, and that $\sigma$ and $\tau$ are scalar multiples of states. Since $\omega$ is pure, $\sigma=\alpha \omega$ for some real number $\alpha$. From $\sigma\left(V^{*} V\right)=\alpha \omega\left(V^{*} V\right)$ it follows directly that $(\bar{V}, P \bar{V})=\alpha(\bar{V}, \bar{V})$. Since $\mathcal{A} / K$ is dense in $\mathcal{Z}$, it results that for any $x \in Z,(x, P x)=\alpha(x, x)$. If $x$ is set equal, successively, to elements of $\mathcal{S}$ and $\mathcal{S} \perp$, a contradiction is obtained with the assumed proper nonzero character of $\delta$.

Now we show, conversely, that a normalizing function of an irreducible representation is pure. Assume that the state $\omega$ is a normalizing function for the irreducible representation $\phi$ of $\mathcal{A}$ on $Z$, and that $\omega=\alpha \sigma+\beta \tau$, where $\sigma$ and $\tau$ are states of $\mathcal{A}$. Define a new inner product on $A / K$ as follows: $[\bar{U}, \bar{V}]=\sigma\left(V^{*} U\right)$. Clearly $[\bar{U}, \bar{V}]$ can be extended, by uniform continuity as above, to an inner product on $Z$ (which is positive semi-definite but not necessarily positive definite). By the Riesz representation theorem for linear functionals, $[\bar{U}, \bar{V}]$ has the form $(B \bar{U}, \bar{V})$, where $B$ is a self-adjoint bounded linear operator on $Z$. Now we show that $B$ commutes with $\phi(W)$ for every $W$ in $A$,

$$
\begin{aligned}
(B \phi(W) \bar{U}, \bar{V}) & =\sigma\left(V^{*} W U\right)=\sigma\left(\left(W^{*} V\right)^{*} U\right)=\left(B \bar{U}, \phi\left(W^{*}\right) \bar{V}\right) \\
& =\left(B \bar{U}, \phi\left(W^{*}\right) \bar{V}\right)=(\phi(W) B \bar{U}, \bar{V}) .
\end{aligned}
$$

It is easy to verify that the range of any projection in the spectral resolution of $B$ is invariant under $\phi(W), W \in \mathcal{C}$, and by the irreducibility of $\phi, B=\beta I$. This shows that $\sigma=\beta \omega-$ that is, $\omega$ is pure-and thereby completes the proof.

4. Existence of irreducible representations. Next we show that a $C^{*}$-algebra has a complete set of irreducible representations, or alternatively, a complete collection of pure states. This result follows from Theorem 1 combined with a theorem of Krein, Milman, and Shmulyan [4] which asserts that a convex set, in the conjugate space of a Banach space, which is compact in the weak (neighborhood) topology, is the weak compactification of the convex core of its extreme points, an extreme point being one which is not an inner point of a segment contained in the set. A pure state is clearly precisely an extreme point of the set of all states, and the proof is largely concerned with showing that this set satisfies the hypothesis of the theorem just quoted.

THEOREM 2. A $C^{*}$-algebra has a complete set of irreducible representations. 
First we observe that it is sufficient to give the proof in the case of an algebra which contains the identity operator. For if $\mathcal{A}$ does not contain $I$, the algebra $\mathcal{A}^{\prime}$ generated (algebraically) by $I$ and $\mathcal{A}$ is readily seen to be a $C^{*}$-algebra; it is clear that an irreducible representation of $\mathcal{A}^{\prime}$ induces an irreducible representation of $\mathcal{A}$. Now set $\Omega$ for the collection of all states of the $C^{*}$-algebra $A$, which is assumed to contain $I$. If $\omega_{\nu}$ is a directed system of elements of $\Omega$ which converges weakly to a linear function $\sigma$ on $\mathcal{A}$, it is clear that $\sigma\left(U^{*}\right)$ is the complex conjugate of $\sigma(U)$, that $\sigma\left(U^{*} U\right) \geqq 0$, and that $\sigma(I)=1$. Hence $\sigma$ is a state of $\mathcal{A}$, and therefore $\Omega$ is complete. Also $\Omega$ is totally bounded because for $\omega \in \Omega,|\omega(U)|^{2} \leqq \omega\left(U^{*} U\right) \leqq\|U\|^{2}$, showing that $\Omega$ is contained in the (compact) unit sphere of the conjugate space of $\mathcal{A}$. It follows that $\Omega$ is compact.

Next we show that $A$ has a complete set of pure states. The collection II of pure states of $\mathcal{A}$ is clearly the same as the collection of extreme points of $\Omega$. If the collection were not complete, there would exist a nonzero element $U$ of $A$ such that $\omega(U)=0$ for $\omega \in \Pi$. It would then follow from the theorem of Krein, Milman, and Shmulyan, that $\omega(U)=0$ for all $\omega \in \Omega$. In particular, $(U x, x)=0$ for every $x$ in the original Hilbert space, which implies $U=0$, a contradiction.

It is trivial to conclude, utilizing Theorem 1 , that $A$ has a complete set of irreducible representations.

5. Unitary representations of groups. In the present section the theorem of Gelfand and Rykov, quoted in the introduction, is proved by applying Theorem 2 to an operator algebra $\mathcal{A}$ which is defined in terms of Haar measure on the group. From representations of $\mathcal{A}$ are derived strongly continuous unitary representations of the group, such a representation being defined in Definition 5 .

DEFINITION 5. A unitary representation of a topological group $G$ is a mapping $\Phi$ on the group to the collection of unitary operators on a Hilbert space $\mathcal{H}$, such that $\Phi(a b)=\Phi(a) \Phi(b)$ for general elements of the group $a$ and $b$, and $\Phi(e)=I$, where $e$ is the group identity. Such a representation is called strongly continuous in case $\Phi(a) x$ is a continuous function on $G$ to $\mathfrak{H C}$ for every fixed $x \in \mathcal{H}$. It is irreducible if $\mathfrak{H C}$ contains no proper closed invariant subspace other than the nullspace.

We mention that the weakly closed operator algebra generated by the faithful unitary representation of $G$ obtained by Haar is not suitable for use as $\mathcal{A}$. While a complete set of irreducible representations of $G$ can be obtained from that algebra, these representations may fail (except when $G$ is compact) to be continuous-such failure is in evi- 
dence when $G$ is the additive group of the reals. Actually $\mathcal{A}$ is taken to be a certain weakly dense subalgebra of the former algebra, and is the uniform closure of an operator algebra isomorphic to the group algebra.

Corollary 2.1 (Gelfand and Rykov). A locally compact group has a complete set of irreducible, strongly continuous, unitary representations on Hilbert spaces.

Let $L_{2}$ be the Hilbert space of complex-valued functions on the group $G$ which are square-integrable relative to (left) Haar measure, the inner product of the function $p$ with the function $q$ being defined as $\int_{G P}(a)(q(a))^{c} d a, d a$ referring to Haar measure. Putting $L_{1}$ for the space of complex-valued functions on $G$ which are integrable relative to Haar measure, we define $U_{f}$ for $f \in L_{1}$ as the following operator on $L_{2}$ :

$$
\left(U_{f} p\right)(b)=\int_{G} f(b) p\left(b^{-1} a\right) d b,
$$

for $p \in L_{2} . U_{f}$ is a bounded operator of bound not greater than $\int_{G}|f(a)| d a$, and it is straightforward to verify that (a) $\left(U_{f}\right)^{*}=U_{f^{*}}$, where $f^{*}(a)=\delta(a)\left(f\left(a^{-1}\right)\right)^{c}, \delta(a)$ being defined by the equation $d a^{-1}=\delta(a) d a$; (b) $U_{f+o}=U_{f}+U_{g}$, (c) $U_{\alpha f}=\alpha U_{f}$ if $\alpha$ is a complex number, (d) $U_{f o}=U_{f} U_{g}$ where $f g$ is defined by:

$$
(f g)(a)=\int_{G} f(b) g\left(b^{-1} a\right) d b .
$$

(Compare [7]. We shall have no occasion to consider the binary operation of ordinary, pointwise, multiplication of functions on $G$, and therefore do not need to introduce any special symbols for the operation just defined.) Calling the collection of operators of the form $U_{f}$ (with $\left.f \in L_{1}\right) \mathcal{A}_{0}$, it follows that the uniform closure $\mathcal{A}$ of $\mathcal{A}_{0}$ is a $C^{*}$-algebra.

The proof shows incidentally that for any normal representation $\phi$ of $\mathcal{A}$, there is a strongly continuous representation $a \rightarrow B_{a}$ of $G$ such that for $f \in L_{1}$,

$$
\phi\left(U_{f}\right)=\int_{G} f(a) B_{a} d a,
$$

where the integral is appropriately defined. Actually the main burden of the proof is to show, utilizing an extension of a method in [7], that this is the case. Corollary 2.1 then follows directly from a known result in the theory of vector-valued integrals. 
Now let $\omega$ be a state of $\mathcal{A}$ and let $\phi, Z$, and so on, be as in the proof of Theorem 1. For $a \in G, R_{a}$ is defined to be the operator on $L_{2}$ which takes $p$ into $p_{a}$, where $p_{a}(b)=p\left(a^{-1} b\right)$ for $p \in L_{2}$, and $A_{a}$ is defined to be the operator on $A / K$ given by $A_{a} \bar{W}=\eta\left(R_{a} W\right), W \in \mathcal{A}$. To show that this definition is unique, we first observe that $R_{a} \mathcal{A}_{0} \subset \mathcal{A}_{0}$, because $R_{a} U_{f}=U_{g}$, where $g(b)=f\left(a^{-1} b\right)$. Since $R_{a}$ is unitary it follows that $R_{a} \mathcal{A} \subset \mathcal{C}$; thus, $R_{a} W$ is actually an element of $\mathcal{A}$. Secondly, if $W_{1} \equiv W_{2}$ modulo $K$, then $R_{a} W_{1} \equiv R_{a} W_{2}$ modulo $K$. For, setting $X=W_{1}-W_{2}$, we have $X \in K$, that is, $\omega(V X)=0$ for all $V \in \mathcal{A}$. Clearly $\omega\left(V\left(R_{a} X\right)\right)=\omega\left(\left(V R_{a}\right) X\right)$, and it is easy to verify that $V R_{a} \in \mathcal{A}$, whence $\omega\left(V\left(R_{a} X\right)\right)=0$, for $V \in \mathcal{A}$. It follows that $R_{a} X \in K$ and $R_{a} W_{1} \equiv R_{a} W_{2}$ modulo $K$.

It is plain that $\left(A_{a} \bar{W}, A_{a} \bar{W}\right)=\omega\left(W^{*} W\right)=(\bar{W}, \bar{W})$, that is, $A_{a}$ is isometric, and therefore uniformly continuous, on $A / K$. It follows that $A_{a}$ can be (uniquely) extended to an isometric operator $B_{a}$ defined on Z. It is plain that $A_{a} A_{a^{-1}}$ is the identity on $A / K$. Hence $B_{a} B_{a^{-1}}$ is the identitv on $Z$, implying that the range of $B_{a}$ is $Z$, and therefore $B_{a}$ is unitary.

Now $B_{a}$ is evidently a representation of $G$. Next we show that this representation is strongly continuous. For this purpose we note that (see [8]), if $f \in L_{1}$, the mapping $a \rightarrow f_{a}$, where $f_{a}(b)=f\left(a^{-1} b\right)$, is continuous on $G$ to $L_{1}$. Hence the mapping $a \rightarrow R_{a} W$ is continuous on $G$ to $A$, if $W \in \mathcal{A}_{0}$. If $W$ is any element of $\mathcal{A}$ we choose $W_{n} \in \mathcal{A}$ such that $\left\|W_{n}-W\right\| \rightarrow 0$; then $\left\|R_{a} W_{n}-R_{a} W\right\| \rightarrow 0$ uniformly in $a$, showing that the mapping is continuous for $W \in \mathcal{C} A$. Now

$$
\begin{aligned}
\left(\left(B_{a}-B_{b}\right) \bar{W},\left(B_{a}-B_{b}\right) \bar{W}\right) & =\left(\eta\left(R_{a} W\right)-\eta\left(R_{b} W\right), \eta\left(R_{a} W\right)-\eta\left(R_{b} W\right)\right) \\
& =\left(\eta\left(\left(R_{a}-R_{b}\right) W\right), \eta\left(\left(R_{a}-R_{b}\right) W\right)\right) \\
& \leqq\left\|\left(R_{a}-R_{b}\right) W\right\|^{2} .
\end{aligned}
$$

Hence $B_{a} \bar{X}$ is continuous on $G$ to $Z$; and $A / K$ being dense in $Z$ and $B_{a}$ being unitary, it follows, by another application of the principle that a uniform limit of continuous functions is continuous, that $B_{a} x$ is a continuous function on $G$ to $Z$ for every $x \in Z$.

To show that the group representation is irreducible when the same is true of the representation of $\mathcal{A}$ we observe that the following integral, whose integrand has its values in a Banach space $\Upsilon$, exists in the sense of Bochner [1] (as extended by Dunford and Pettis from the case of functions on a Euclidean space relative to Lebesgue measure to the case of functions on an arbitrary decomposable measure space): 


$$
\int_{H} y(a) f(a) d a,
$$

where $f \in L_{1}, y(a)$ is a bounded continuous function on $G$ to $\Upsilon$, and $H$ is a countable sum of compact subsets of $G$ such that $\int_{G-H}|f(a)| d a=0$. For such an integral exists provided: (a) the norm of the integrand is majorized by an integrable real-valued function-which is plainly the case here; (b) there exists a sequence of finitely-valued functions (that is, functions whose range of values is finite and whose sets of constancy are measurable) on $G$ to $\Upsilon$ which converge almost everywhere (a.e.) to the integrand-which is now shown to be valid in the present case. Let $f_{n}(a), n=1,2, \cdots$, be a sequence of finitely-valued functions on $G$ to the complex numbers which converge a.e. to $f(a)$. Let $C_{n}, n=1,2, \cdots$, be a monotone increasing sequence of compact subsets of $G$ whose union is $H$. Now cover $C_{n}$ by the compact sets $D_{n 1}, \cdots, D_{n m}$, on each of which the oscillation of $y(a)$ is less than $n^{-1}$ (each set $D_{n j}$ being contained in $C_{n}$ ). Let $a_{n j}$ be any point of $D_{n j}$, and define $y_{n}(a)$ as follows: (a) for $a \in D_{n 1}, y_{n}(a)=y\left(a_{n 1}\right)$, (b) for $a \in D_{n(j+1)}-U_{k \leqq j} D_{n k}, y_{n}(a)=y\left(a_{n(j+1)}\right)$; (c) $y_{n}(a)=0$ if $a \in G-C_{n}$. It is clear that $\lim y_{n}(a) f_{n}(a)=y(a) f(a)$ a.e., and that $y_{n}(a) f_{n}(a)$ is finitely-valued.

In particular, the integral exists in case either $y(a)=R_{a} W$ or $y(a)=R_{a} p$, with $p \in L_{2}$ (see [7] for a proof that the mapping $a \rightarrow p_{a}$, where $p_{a}(b)=p\left(a^{-1} b\right)$, is continuous on $G$ to $\left.L_{2}\right)$. Now $\int_{H} R_{a} p f(a) d a$ $=U_{f} p$, because for any $q \in L_{2}$

$$
\int_{G}\left(U_{f} p\right)(b)(q(b))^{c} d b=\int_{G}\left(\int_{H} R_{a} p f(a) d a(b)\right) \eta(q(b)) d b,
$$

by the Fubini theorem and the fact that continuous linear operations commute with integration. If we set $p=W r$, and observe that $\int_{H} R_{a} W r f(a) d a=\left(\int_{H} R_{a} W f(a) d a\right) r$, it follows that $U_{f} W=\int_{H} R_{a} W f(a) d a$. Since the operation $X \rightarrow \bar{X}$ is a continuous linear operation on $\mathcal{A}$ to $A / K, \phi\left(U_{f}\right) \bar{W}=\int_{H} B_{a} \bar{W} f(a) d a$; and it is easily shown, from the fact that $\int_{H} B_{a} x f(a) d a$ exists for $x \in Z$ and is a continuous function of $x$, that $\phi\left(U_{f}\right) x=\int_{H} B_{a} x f(a) d a$ for arbitrary $x \in Z$. Now $\int_{H} B_{a} x f(a) d a$, like any Bochner integral, is in the span of the range of values of the integrand, that is, in the span of $\left[B_{a} x \mid a \in G\right]$. Hence a closed linear manifold which is invariant under the group representation is invariant under $\phi\left(U_{f}\right)$ for every $f \in L_{1}$, and therefore invariant under the representation of $\mathcal{A}$.

\section{The completeness of irreducible representations in quantum}


theory. In this section we prove a theorem which implies that for the computational purposes of quantum theory it is sufficient to consider only those representations of a $C^{*}$-algebra (which in quantum theory is the algebra generated by the observables) which are irreducible. A special case of the theorem asserts that the spectrum of a self-adjoint operator in a $C^{*}$-algebra is determined by the collection of spectra of the operator in the irreducible representations of the algebra. In practice only the irreducible representations are used, in general. Our result shows that this limitation to irreducible representations is valid for an arbitrary physical system which is describable by an algebra of operators on a Hilbert space (in the case of the Schrödinger-Heisenberg non-relativistic theory this fact had been established by special considerations by von Neumann [5]).

In order to treat the simultaneous analysis of a commutative collection of SA operators, as well as the analysis of a single operator, we introduce the concept of observation of an algebra.

Definition 6. An observation of a $C^{*}$-algebra is a state $\omega$ such that $\omega(U V)=\omega(U) \omega(V)$ for all $U$ and $V$ in the algebra.

To give an interesting example of an observation, let $A$ be the $C^{*}$-algebra generated by a bounded SA operator $T$, and let $\alpha$ be an arbitrary point in the spectrum of $T$. It is plain from the spectral theorem that every element of $\mathcal{A}$ has the form $f(T)$, where $f$ is a complexvalued function which is defined and continuous on the spectrum of $T$, and conversely, if $f$ is any such function, and if $f(0)=0$, then $f(T) \in \mathcal{A}$. Now define a linear functional $\omega$ on $\mathcal{A}$ as follows: $\omega(f(T))=f(\alpha)$. It is easy to verify that $\omega$ is an observation. On the other hand, every observation of $A$ is of the preceding form. This follows from a known result about an algebra of all continuous functions on a compact Hausdorff space, when it is observed that $\mathcal{A}$ is isomorphic with the algebra of all continuous functions on the spectrum of $T$, except that only functions vanishing at 0 are included if 0 is in the spectrum of $T$ (it is trivial to modify the known result so as to apply to this case also).

This example shows that the following result is a special case of Corollary 2.2. If $\alpha$ is in the spectrum of a self-adjoint element $U$ of a $C^{*}$-algebra $\mathcal{A}$, then there exists an irreducible representation $\phi$ of $A$ and a nonzero element $x$ of the space on which $A$ is represented such that

$$
\phi(U) x=\alpha x .
$$

Thus there is in a sense always a wave function for a point in the spectrum of $U$, whether it is in the point or continuous spectrum; this 
wave function, naturally, will usually not be in the space on which $U$ operates. Corollary 2.2 has the obvious partial converse, that if $\phi$ is a normal representation of a $C^{*}$-algebra $A$, then every observation $\omega^{\prime}$ on the $C^{*}$-algebra $\phi(\mathcal{B}), \mathcal{B}$ being a commutative $C^{*}$-subalgebra of $\mathcal{A}$, defines an observation $\omega$ on $\mathbb{B}$, by the equation $\omega(U)=\omega^{\prime}(\phi(U))$.

Corollary 2.2. If $\tau$ is an observation of a $C^{*}$-subalgebra $\mathcal{B}$ of a $C^{*}$-algebra $\mathcal{A}$, then there exists an irreducible representation $\phi$ of $\mathcal{A}$ and a nonzero element $x$ of the space on which $A$ is represented such that for $U \in \mathfrak{B}$,

$$
\phi(U) x=\tau(U) x .
$$

First we prove a lemma of some general interest.

Lemma. An observation of a $C^{*}$-subalgebra of a $C^{*}$-algebra is pure on the subalgebra and coincides on the subalgebra with a pure state of the algebra.

Let $\tau$ be an observation of the $C^{*}$-subalgebra $B$ of the $C^{*}$-algebra $\mathbb{B}$. If the notation of the proof of Theorem 1 is utilized, with $A$ replaced by $\mathcal{B}$, it is plain that for arbitrary $U$ and $V$ in $\mathcal{B}$,

$$
\tau(U V)=(\phi(U V) z, z)=\left(\phi(V) z, \phi\left(U^{*}\right) z\right) .
$$

On the other hand, $\tau(U V)=\tau(U) \tau(V)=(\phi(U) z, z)(\phi(V) z, z)$. Now by Schwarz' inequality,

$$
\begin{aligned}
\left(\phi(V) z, \phi\left(U^{*}\right) z\right) & \leqq(\phi(V) z, \phi(V) z)^{1 / 2}\left(\phi\left(U^{*}\right) z, \phi\left(U^{*}\right) z\right)^{1 / 2} \\
& =\left(\phi\left(V^{*} V\right) z, z\right)^{1 / 2}\left(\phi\left(U U^{*}\right) z, z\right)^{1 / 2} \\
& =|\tau(U) \tau(V)| .
\end{aligned}
$$

Since the equality holds for Schwarz' inequality only when the vectors involved are proportional, it follows that $\phi(V) z$ and $\phi\left(U^{*}\right) z$ are proportional. In other words, the representation space $Z$ is one-dimensional. This implies, clearly, that $\phi$ is irreducible. Hence, applying Theorem 1, the normalizing function $\tau$ of $\phi$ is pure.

To prove the remainder of the lemma we show that it is sufficient to consider the case in which $I \in \mathcal{B}$. Because if $\mathbb{B}$ does not contain $I$, the observation $\tau$ of $B$ may be extended to an observation $\tau^{\prime}$ of the $C^{*}$-algebra $\mathbb{B}^{\prime}$ generated by $\mathcal{B}$ and $I$ as follows: for $U \in \mathcal{B}, \tau^{\prime}(\alpha I+U)$ $=\alpha+\tau(U)$. (As noted above, the algebra generated algebraically by $B$ and $I$ is a $C^{*}$-algebra.) It is clear that $\tau^{\prime}(V W)=\tau^{\prime}(V) \tau^{\prime}(W)$ for $V$ and $W$ in $\mathcal{B}^{\prime}$. In order to complete the verification that $\tau^{\prime}$ is a state it is plainly sufficient to show that $\tau^{\prime}(\alpha I+U) \geqq 0$ if $\alpha I+U$ is SA and not less than 0 . Now $\alpha I+U \geqq 0$ implies that $\alpha I+U=V^{2}$ with $V$ SA 
and in $\mathcal{B}^{\prime}$ and hence $\tau^{\prime}(\alpha I+U)=\left(\tau^{\prime}(V)\right)^{2} \geqq 0$. Assuming that the lemma had been proved for the case $I \in B$, it would follow that $\tau^{\prime}$ coincides on $\mathcal{B}^{\prime}$ with a pure state $\omega^{\prime}$ of $\mathcal{A}^{\prime}$, where $\mathcal{A}^{\prime}$ is the $C^{*}$-algebra generated by $\mathcal{A}$ and $I$. Putting $\psi^{\prime}$ for a normal representation of $\mathcal{A}^{\prime}$ with normalizing function $\omega^{\prime}(U)=\left(\psi^{\prime}(U) x^{\prime}, x^{\prime}\right)$, it follows that $\psi^{\prime}$ is irreducible, and moreover the representation $\psi$ obtained by restricting $\psi^{\prime}$ to $A$ is irreducible and has the normalizing function $\omega$, where $\omega$ is obtained by restricting $\omega^{\prime}$ to $\mathcal{A}$. Hence $\omega$ is pure. Thus $\tau$ coincides on $\mathcal{B}$ with the pure state $\omega$ of $\mathcal{A}$.

Now it is assumed that $I \in \mathcal{B}$. Put $\mathcal{A}_{0}$ and $\mathbb{B}_{0}$ for the collection of $\mathrm{SA}$ elements of $\mathcal{A}$ and $B$, respectively. We show next, by the method of proof of the Hahn-Banach theorem, that $\tau$ coincides on $\mathcal{B}_{0}$ with a real-valued linear function $\chi_{0}$ on $A_{0}$ which is non-negative on positive semi-definite elements of $\mathcal{A}_{0}$. Let $U$ be an arbitrary element of $\mathcal{A}_{0}$ which is not in $\mathcal{B}_{0}$. Put $P=\left[V \mid V \in \mathcal{A} \mathcal{A}_{0}, V \geqq U\right]$ and $2=[V \mid V \in \mathcal{A}$, $V \leqq U]$. Since $-\|U\| I \leqq U \leqq\|U\| I$, neither $\mathcal{P}$ nor 2 is empty. Defining $\gamma_{-}=$L.U.B. $v \in Q^{\tau}(V)$ and $\gamma_{+}=$G.L.B. $v \in \mathcal{P} \tau(V)$, it is clear that $\gamma_{-} \leqq \gamma_{+}$. Let $\gamma$ be a number such that $\gamma_{-} \leqq \gamma \leqq \gamma_{+}$. Now we define $\chi_{0}$ on the space $\mathcal{B}_{0}{ }^{+}$spanned by $\mathcal{B}_{0}$ and $U$ as follows: for $V \in \mathcal{B}_{0}$, $\chi_{0}(\alpha U+V)=\alpha \gamma+\tau(U)$. It is obvious that $\chi_{0}$ is linear on $\mathcal{B}_{0}{ }^{+}$. To see that $\chi_{0}$ is non-negative on positive semi-definite elements of $\mathcal{B}_{0}^{+}$, note that if $\alpha U+V \geqq 0$, and if $\alpha>0$, then $U \geqq-\alpha^{-1} V,-\alpha^{-1} V \in 2$, $\chi_{0}\left(-\alpha^{-1} V\right)=\tau\left(-\alpha^{-1} V\right) \leqq \gamma-\leqq \gamma=\chi_{0}(U)$, and finally, $\chi_{0}(\alpha U+V) \geqq 0$; and a similar proof is valid if $\alpha<0$. It is plain that by transfinite induction we may obtain a function $\chi_{0}$ on $\mathcal{A}_{0}$ with the stated property. It is easy to verify that $\mathcal{A}$ is the (real) linear direct sum of $\mathcal{A}_{0}$ and $i A_{0}$. Hence the definition

$$
\chi(U+i V)=\chi_{0}(U)+i \chi_{0}(V), \quad U \text { and } V \text { in } \mathcal{A}_{0},
$$

extends $\chi_{0}$ to $\mathcal{A}$. It is plain that $\chi$ is a state of $\mathcal{A}$, and that it coincides on $B$ with $\tau$. Thus the class $\Pi$ of states of $\mathcal{A}$ which coincide on $B$ with $\tau$ is nonvacuous.

It is obvious that $\Pi$ is convex. To see that it is compact in the weak neighborhood topology, observe that $\pi(U)$ is a continuous function of $\pi$, for fixed $U$. It was shown in the proof of Theorem 2 that $\Omega$ is compact. Now $\Pi$ is an intersection of closed subsets of $\Omega$ and therefore is itself closed, and hence compact. It follows, if we apply again the theorem of Krein, Milman, and Shmulyan, that $I I$ contains an extreme point $\omega$.

Next we show that $\omega$ is pure. Suppose that $\omega=\alpha \rho+\beta \sigma$ where $\rho$ and $\sigma$ are states of $\mathcal{A}$ and $\alpha$ and $\beta$ are non-negative numbers. Then $\omega(I)=\alpha \rho(I)+\beta \sigma(I)$, which implies $\alpha+\beta=1$. Now we adopt the fol- 
lowing notation for a state of $\mathcal{B}$ which is obtained from a given state of $\mathcal{A}$ by restricting its domain of definition to $\mathcal{B}$ : for $\xi \in \Omega, \xi_{B}$ denotes the state of $\mathcal{B}$ which coincides with $\xi$ on $\mathcal{B}$. It is plain that $\omega_{B}=\alpha \rho_{B}$ $+\beta \sigma_{B}$. But $\omega_{B}=\tau$ and is therefore pure. Hence $\rho_{B}=\sigma_{B}=\tau$. It follows that $\rho$ and $\sigma$ are elements of $\Pi$. On the other hand, $\omega$ is an extreme point of $\Pi$, and hence the equation $\omega=\alpha \rho+\beta \sigma$ implies that $\rho=\sigma=\omega$ that is, $\omega$ is pure. The proof of the lemma is now complete.

To conclude the proof of Corollary 2.2, let $\omega$ be a pure state of $A$ which coincides with $\tau$ on $B$, and let $\phi$ be a normal representation of $A$ with normalizing function $\omega$, and adopt the rest of the notation of the proof of Theorem 1. It is clear that for $U$ and $V$ in $\mathbb{B}$,

$$
\omega\left((U V-\omega(U) V)(U V-\omega(U) V)^{*}\right)=0 .
$$

Hence $U V-\omega(U) V$ maps into zero under the natural mapping on $\mathcal{A}$ to $A / K$, or

$$
\phi(U) \bar{V}=\omega(U) \bar{V} .
$$

The proof is completed by observing that $\bar{V} \neq 0$ for some $V$ in $\mathbb{B}$, because otherwise $\omega\left(V^{*} V\right)=0$ for all $V$ in $\mathcal{B}$, which is evidently not the case.

\section{REFERENCES}

1. S. Bochner, Integration von Funktionen deren Werte die Elemente eines Vektorräumes sind, Fund. Math. vol. 20 (1933) pp. 262-276.

2. I. Gelfand and M. Neumark, On the imbedding of normed rings into the ring of operators in Hilbert space, Rec. Math. (Mat. Sbornik) N. S. vol. 12 (1943) pp. 197-213.

3. I. Gelfand and D. Rykov, Irreducible unitary representations of locally compact groups, Rec. Math. (Mat. Sbornik) N. S. vol. 13 (1943) pp. 301-316. A summary of this article was published in C. R. Acad. Sci. URSS. vol. 42 (1944) pp. 199-201.

4. M. Krein and D. Milman, On extreme points of regular convex sets, Studia Mathematica vol. 9 (1940) pp. 133-137.

5. J. von Neumann, Die Eindeutigkeit der Schrödingerschen Operatoren, Math. Ann. vol. 104 (1931) pp. 570-578.

6. I. E. Segal, The group algebra of a locally compact group, Trans. Amer. Math. Soc. vol. 61 (1947) pp. 69-105.

7. A. Weil, L'intégration dans les groupes topologiques et ses applications, Actualités Scientifiques et Industrielles, No. 869, Paris, 1940.

InSTITUTE For AdVANCED StUdy 